\title{
What is the outcome of thrombolysing stroke patients in Gwent, Wales?
}

\author{
Authors: Amer Jafar, ${ }^{A}$ Pak Tsz Yu Eunice, ${ }^{B}$ Anthony Wijaya, ${ }^{B}$ Aisling Sweeney ${ }^{B}$ and Wan Li Cheah ${ }^{B}$
}

\section{Introduction}

Stroke is the third most common cause of death and a major cause of disability worldwide.

The benefits of thrombolysing ischaemic stroke patients outweigh the risks within the time window of $<4.5$ hours of the symptoms presentation. This study was to analyse the data regarding the outcome of thrombolysing stroke patients in Gwent, after centralising the stroke thrombolysing service in south-east Wales.

\section{Methods}

Retrospective databases during 2016 to 2018 were reviewed for neurological outcome after 3 months in relation to demographics, door-to-computed tomography (CT) time, door-to-needle time, type of stroke and the discharge destination. Univariate and multivariate analyses were performed.

\section{Results}

Of the total 1,748 patients, 262 were excluded as they were diagnosed with brain haemorrhage. Another 87 patients were excluded as they were stroke mimics. We thrombolysed $13.7 \%$ of stroke patients with an average age for both sexes of 72.3 years. The average age for the female patients was 75 years, while that for male patients was 69 years. The average door-to-CT brain time was 38 minutes, while the average door-to-needle time was 85 minutes. The majority of the thromobolysed patients were partial anterior circulation infarct (PACI) $(62.7 \%)$. The majority of patients went home (77.6\%) after thrombolysis with an average length of stay of 8 days in the acute stroke ward. The mortality rate was $17 \%$. More male patients died after thrombolysis than female, but the difference was non-significant. The average Modified Rankin Scale (MRS) for patients who went home was 2.

\section{Conclusion}

This is probably the first report to show independent improvement in outcome of thrombolysing ischaemic stroke in Gwent. With the clinical future strategy of the Aneurin Bevan Health Board, we expect further improvement in the outcome and higher rate

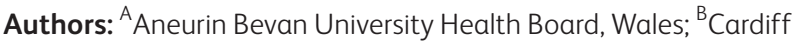
University Medical School, Wales, UK of thrombolysing stroke patients in Gwent. We need to intensify our efforts to engage the primary care doctors and the public to increase the awareness of the thrombolysis service.

\section{Conflict of interest statement}

None declared. 